\title{
A Conceptual Model of the Sense of Presence in Virtual Environments
}

\begin{abstract}
A bstract
This paper proposes a model of interaction in virtual environments which we term the immersion, presence, performance (IPP) model. This model is based on previous models of immersion and presence proposed by Barfield and colleagues and Slater and colleagues. The IPP model describes the authors' current conceptualization of the effects of display technology, task demands, and attentional resource allocation on immersion, presence, and performance in virtual environments. The IPP model may be useful for developing a theoretical framework for research on presence and for interpreting the results of empirical studies on the sense of presence in virtual environments. The model may also be of interest to designers of virtual environments.
\end{abstract}

\section{Introduction}

O ne goal of virtual world designers is to create virtual environments that bring about a sense of presence in participants. Presence in virtual environments has been defined as the degree to which participants feel that they are somewhere other than where they physically are when they experience the effects of a computer-generated simulation (Sheridan, 1992a, 1992b; Barfield \& Weghorst, 1993; Slater \& U soh, 1994; Barfield, Sheridan, Z eltzer, \& Slater, 1995). The goal of this paper is to examine features of two models of presence, one proposed by Barfield and colleagues (Barfield \& $H$ endrix, 1995; Barfield, H endrix, \& Bystrom, 1997; H endrix \& Barfield, 1996a, 1996b), the other proposed by Slater and colleagues (Slater \& Wilbur, 1995; Slater, Linakis, U soh, \& Kooper, 1996), and to combine both models into one overall conceptual model of immersion, presence, and performance. For brevity, we term the new model thel PP mode.

The development of models relating to presence and performance in virtual environments is useful for three

Presence, Vol. 8, N 0. 2, April 1999, 241-244 (C) 1999 by the Massachusetts Institute of Technology reasons. First, models on the sense of presence may form a theoretical framework for research on virtual environments. Such a framework would be useful for determining which factors influence presence and thus contribute to the sense of "being there" in the virtual environment. Second, the framework may help researchers investigate the relationships among immersion, presence, and performance in virtual environments. Third, a model of presence may help designers of virtual worlds select appropriate display features when they design virtual environments.

\section{Models of Immersion, Presence, and Performance}

Slater and Wilbur (1995) distinguish between the concepts of immersion and presence. They categorize immersion in a virtual environment as a quantifiable aspect of display technology, primarily determined by the extent to which displays are

(a) inclusive (the degree to which stimuli from the real world are excluded from the user),

(b) extensive (the number of sensory modalities accommodated by the system),

(c) surrounding (how panoramic the displays are), and

(d) vivid (the resolution of the displays).

The categorization of immersion also posits that an egocentric self-representation oriented around a virtual body in the virtual environment is necessary to achieve a high-level match between proprioception and sensory

\footnotetext{
Karl-Erik Bystrom Woodrow Barfield Claudia Hendrix University of W ashington barfield@vt.edu

Virginia Polytechnic Institute and State U niversity U niversity of Minnesota
} 
data (Slater \& Wilbur, 1995, p. 13). In contrast to immersion, they argue that presence is "a state of consciousness, the [psychological] sense of being in the virtual environment" (Slater \& Wilbur, 1995, p. 14). Slater's model predicts that the higher the level of immersion, the higher the level of presence. Slater and Wilbur also claim that the level of presence is determined not only by the four aspects of displays listed above, but is also mediated by the sorts of sensory information required to perform the task at hand (for example, whether visual or auditory data would be of greater use), and the individual differences in preferences for information displayed in various modalities (for example, whether the user tends to prefer visual or auditory displays of information). Slater and Wilbur make this claim only for presence and not for task performance.

Barfield and colleagues (Barfield \& H endrix, 1995; Barfield, H endrix, \& Bystrom, 1997) have proposed a "spatial fidelity" model of presence that posits that the sense of presence is dependent on the degree to which spatial, auditory, and haptic transformations of objects in a virtual environment are similar to spatial, auditory, and haptic transformations of objects in the real world. The fidelity of the spatial transformations are determined by factors such as the geometric field of view, the display update rate (Barfield \& H endrix, 1995), motion parallax, and depth cues such as linear perspective, aerial perspective, and occlusion. For example, motion parallax is an important aspect of spatial perception that in virtual environments is provided by head tracking; so integrating head tracking into virtual environment systems improves the spatial fidelity of those systems. We propose that display technologies and interaction methods that ensure high-fidelity spatial transformations (such as head tracking to provide motion parallax, and update rates fast enough to provide the illusion of continuous motion within the simulation) will tend to provide high levels of presence.

$\mathrm{H}$ owever, the relationship between immersion, presence, and performance in virtual environments is not straightforward. Clearly, the level of immersion as defined by Slater and Wilbur (1995) can influence performance. F or example, in the performance of a spatial task involving depth judgments, stereopsis should lead both to greater immersion and to superior spatial judgments.
In contrast, the spatial realism model postulates that the spatial fidelity of the virtual environment will enhance performance only if the demands of the task will require that a particular spatial cue (e.g., linear perspective, motion parallax, or aerial perspective) be present in that environment. For example, a task that is not dependent on stereopsis as a depth cue (e.g., judging the separation between images displayed at great distances from the observer) would not benefit from the use of a stereoscopic display, even if stereopsis would increase the level of immersion in the virtual environment. Slater et al. (1996) also argue that a sense of presence in a virtual environment will contribute to user behavior that more closely matches real-world behavior. Thus, performance will be improved if the closer matching of behavior would be beneficial. We hypothesize that presence itself doesn't necessarily facilitate or hinder performance, but that having some sense of presence in an environment is a necessary condition for performance to occur. We also postulate that the nature of the task itself may also indirectly influence the level of presence, as a particularly engaging task may lead the user to allocate more attentional resources to the virtual environment, thus bringing about a greater sense of presence. Clearly, whether the display technology used to produce virtual environments will assist task performance will be dependent on the type of task to be performed and the level of immersion. Slater and colleagues further propose that immersion has two additional components, 1) the "extent of the match between the display's sensory data and the internal representation systems and subjective world models that are typically employed by the participant," and 2) the "extent of the relationship between the display technology capabilities and task requirements" (Slater and Wilbur, 1995; Slater and U soh, 1994; Slater, Linakis, U soh, and Kooper, 1996).

\section{An Integrated Model of Immersion, Presence, and Performance}

Figure 1 shows a block diagram representing our current conceptualization of the relationship between immersion, presence, and performance. We refer to this 
model as the I mmersion, Presence, Performance (IPP) model of interaction in virtual environments. The first two components of the model describe the physical characteristics of the virtual environment system itself. The first component represents various types of display technology, also known as "enabling technology," used to produce virtual environments. This enabling technology leads to the second component of the model, Slater's concept of immersion. As noted, this represents quantifiable features of the display technology, such as the resolution of the visual display, or the degrees of freedom associated with an input device or position sensor. The physical characteristics of the system contribute to the third component of the model, the box labeled "sensory fidelity." This component represents the impact of immersion (i.e., features of display technology) on the fidelity of the sensory information displayed to the participant in the virtual environment. M ore specifically, sensory fidelity is the degree to which the display and transformation of spatial, auditory, and haptic information in a virtual environment is similar to the display and transformation of sensory information in the real world. For example, a display with head tracking will be more immersive than a nonhead-tracked display, and will result in a higher level of sensory fidelity because motion parallax is supported.

The subsequent components of the model represent the interactions of the participant with the virtual environment, the objects in it, and the actions the user performs in the virtual environment. I n order to experience and interact with the virtual environment, the participant must allocate attentional resources to the objects and events within the environment. The requirements of the tasks that the user must perform will influence the amount of attentional resources that are allocated to the virtual environment. If the participant allocates sufficient attentional resources to the virtual environment, and if there is a sufficient degree of sensory fidelity, the participant may "suspend disbelief," and view the virtual environment as an actual place, thereby developing a sense of presence in the virtual environment. We postulate that experiencing a sense of presence in the virtual environment is a necessary condition for performance to occur. Furthermore, the nature of the task and user actions may

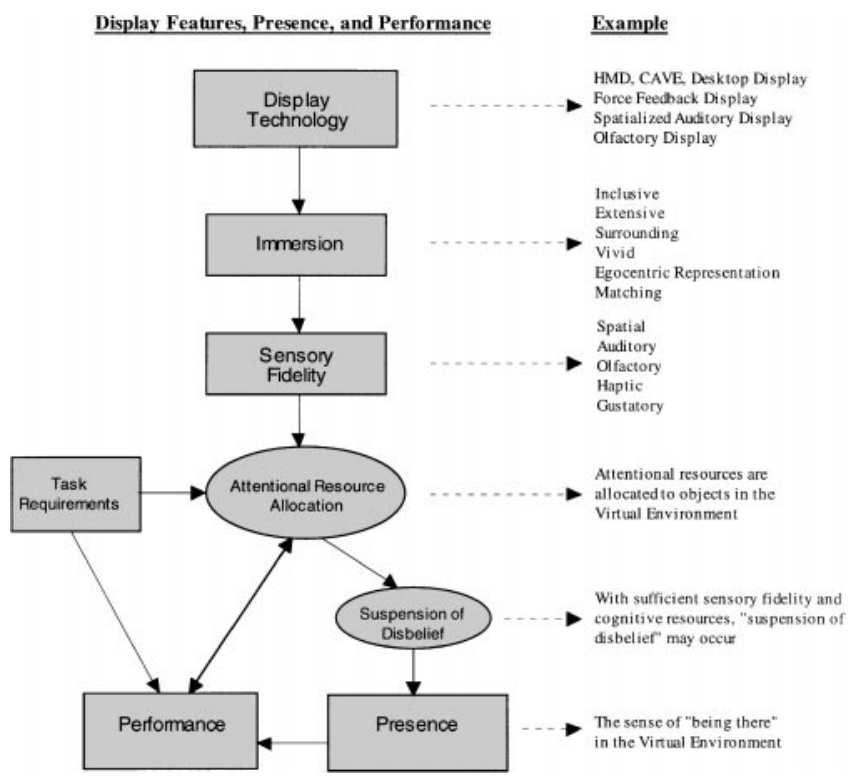

Figure 1. Diagram of a model on immersion, presence, and performance.

also indirectly influence the level of presence (as indicated by the feed back loop to the box labeled "attentional resource allocation"), as a particularly engaging task may lead the user to allocate more attentional resources to the virtual environment, thus bringing about a greater sense of presence. This feedback loop is reminiscent of the interaction between task, user ability, and actions proposed in the Csikszentmihalyis' flow theory (C sikszentmihalyi \& Csikszentmihalyi, 1988), and is similar to the perception/ action coupling that Zahorik and J enison (1998) argue is necessary to experience a sense of presence. Finally, the nature of the task, the level of attention the user gives to the task, and the sense of presence may all affect the quality of performance on the task.

The IPP model attempts to present a conceptual framework for examining user behavior in virtual environments. U nlike purely empirical models, it does not attempt to predict specific user experiences based on parameters in virtual environments. Rather, it presents a general framework for posing questions. H owever, the integrated model is predictive to a degree; for example, the model indicates that, with increasing levels of sensory fidelity and immersion, there should also be equal 
levels of presence, while improving the enabling technology in the virtual environment should improve the sense of presence and may improve task performance depending upon the requirements of the task. The reader should note that we view this as a working model of immersion, presence, and performance. Research on these issues is still in a relatively immature stage, and, as we learn more about interaction in virtual environments, we hope to refine or correct this model. We also believe that this model is not, and should not be considered, a definitive model of interaction in virtual environments. D epending on the goal of the researcher, this model or a different model may be called for. Finally, we hope that this model can be a useful tool for continuing those investigations.

\section{Acknowledgments}

This work was supported in part by the Air Force $O$ ffice of Scientific Research (Contract 92-N L-225 and I N ST PRO P N O : 78216), the $\mathrm{N}$ ational Science Foundation (D M C-8857851, CDA-8806866), and by the Center for Research in L earning, Perception, and Cognition and the $\mathrm{N}$ ational Science Foundation's Program in Visual Perception and M otor Control (GER 9454163).

\section{References}

Barfield, W., \& Weghorst, S. (1993). The sense of presence within virtual environments: a conceptual framework. In G . Salvendy and M. Smith (Eds.), H uman Computer I nteraction: Softwareand $\mathrm{H}$ ardware Interfaces. (pp. 699-704). Elsevier Publisher.

- - , H endrix, C. (1995). The effect of update rate on the sense of presence in virtual environments. Virtual R eality: R esearch, D evel opment, A ppli cations, 1(1), 3-15.
- - Sheridan, T., Zeltzer, D ., \& Slater, M . (1995). Presence and performance within virtual environments. In W. Barfield and T. Furness III (Eds.), Virtual Environmentsand Advanced I nterfaceD esign. (pp. 473-513). O xford U niversity Press.

- - , H endrix, C., \& Bystrom, K. (1997). Visualizing the structure of virtual objects using desktop virtual reality displays. Virtual R eality A nnual I nternational Symposium '97, 114-119.

C sikszentmihalyi, M ., \& C sikszentmihalyi, I. S., (Eds.). (1988). O ptimal experience: Psychological studies of flow in consciousness. Cambridge: C ambridge $U$ niversity Press.

H endrix, C., \& Barfield, W. (1996a). Presence within virtual environments as a function of visual display parameters. Pres ence: Teleoperatorsand Virtual Environments, 5 (3), 274289.

- - , \& - - . (1996b). The sense of presence within auditory virtual environments. Presence: Tel eoperators and Virtual Environments, 5 (3), 290-301.

Sheridan, T. B. (1992a). M usings on telepresence and virtual presence. Presence: Teleoperatorsand Virtual Environments, 1 (1), 120-125.

- - . (1992b). D efining our terms. Presence: Teleoperatorsand Virtual Environments, 1 (2), 272-274.

Slater, M ., \& Wilbur, S. (1995). Through the looking glass world of presence: A framework for immersive virtual environments. In M. Slater (Ed.), FIVE '95 Framework for I mmersiveVirtual Environments Q M W U niversity of L ondon.

- - , \& U soh, M . (1994). Representations systems, perceptual position, and presence in immersive virtual environments. Presence: Teleoperatorsand Virtual Environments, 2 (3), 221-233.

- - , Linakis, V., U soh, M ., \& Kooper, R. (1996, July). Immersion, presence, and performance in virtual environments: An experiment in tri-dimensional chess. Proceedings of VR ST '96, H ong Kong.

Zahorik, P., \& Jenison, R. L. (1998). Presence as being-in-theworld. Presence: Teleoperatorsand Virtual Environments, 7 (1), 78-89. 